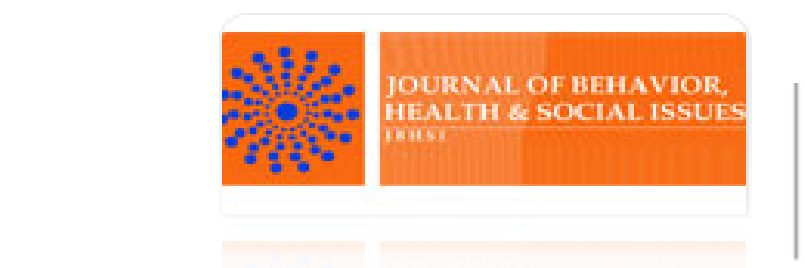

Journal of Behavior, Health \& Social Issues

ISSN: 2007-0780

jcpedro@unam.mx

Asociación Mexicana de Comportamiento y Salud, A. C.

México

Martínez-Munguía., Carlos; Quintana-Rodríguez., Carmen; Ortiz-Rueda., Gerardo GÉNERO Y CONDUCTAS DE RIESGO PARA LA SALUD: UNA APROXIMACIÓN DESDE UN PUNTO DE VISTA INTERCONDUCTUAL

Journal of Behavior, Health \& Social Issues, vol. 6, núm. 2, noviembre, 2014, pp. 57-69 Asociación Mexicana de Comportamiento y Salud, A. C.

Distrito Federal, México

Disponible en: http://www.redalyc.org/articulo.oa?id=282235731005

Cómo citar el artículo

- Número completo

- Más información del artículo

- Página de la revista en redalyc.org

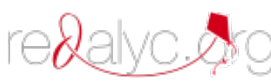

Sistema de Información Científica

Red de Revistas Científicas de América Latina, el Caribe, España y Portugal Proyecto académico sin fines de lucro, desarrollado bajo la iniciativa de acceso abierto 


\section{GÉNERO Y CONDUCTAS DE RIESGO PARA LA SALUD: UNA APROXIMACIÓN DESDE UN PUNTO DE VISTA INTERCONDUCTUAL}

\section{GENDER AND RISK-TAKING BEHAVIORS RELATED TO HEALTH: AN INTERBEHAVIORAL APPROACH}

\author{
Carlos Martínez-Munguía. \\ Carmen Quintana-Rodríguez. \\ Gerardo Ortiz-Rueda. \\ Centro de Estudios e Investigaciones en \\ Comportamiento - Universidad de Guadalajara, \\ Guadalajara, México.
}

\author{
Recibido: Septiembre 2, 2013 \\ Revisado: Mayo 5, 2014 \\ Aceptado: Julio 10, 1014 \\ Autor responsable Carlos Martínez-Munguía Maestro en Ciencias, \\ email: carmtzQcencar.udg.mx Guadalajara, Jal. México. Proyecto \\ financiado por COECYTJAL clave: 2009-05-125691.
}

El abordaje multidisciplinario del género se ha estancado por la falta de precisión y claridad conceptual, incluyendo lo que desde una perspectiva ryleana se conoce como errores categoriales entre algunos conceptos disciplinares que intentan dar cuenta del fenómenos, es decir, se toman categorías que corresponden a un determinado ámbito de acción (i.e., psicológico) y se pretende extrapolar a otro (i.e., sociología). Se plantea la pertinencia de delimitar claramente las distintas aristas del estudio del género. Desde la psicología interconductual, se enfatiza el ajuste individual a las convenciones de género, sugiriendo que el análisis de los segmentos de interacción, entre género y conductas de riesgo para la salud, implica la identificación de sus elementos constituyentes y las relaciones situacionales concretas que demarcan un determinado segmento de análisis: macrocontingencias (i.e., convenciones, valores, creencias); y microcontingencias (i.e., situación específica, personas, factores disposicionales). Se concluye que la implementación del análisis aquí propuesto, puede facilitar, tanto en la detección de conductas de riesgo, como en la articulación de estrategias efectivas para su prevención.

Palabras clave: Género, conductas de riesgo, salud, psicología interconductual, jóvenes. 
Martínez-Munguía, Quintana-Rodríguez \& Ortiz-Rueda

Abstract

The multidisciplinary approach of gender has been stalled by a lack of precision and conceptual clarity, including category errors as they are known from a rylean perspective, taking concepts that belongs to a given scope (i.e. psychology) trying to extrapolate them to analyze a phenomena that belongs to another area of knowledge (i.e. sociology). We argue in favor of clarity defining the different facets of the study of gender. An interbehavioral approach emphasizes individual adjustment to the conventions of gender, suggesting that the analysis of the interaction between gender and risk-taking behaviors related to health should involve identifying specific situational elements and relationships: macrocontingencies (i.e. conventions, values, beliefs) and microcontingencies (i.e. specific situation, people, dispositional factors). We conclude that the implementation of the analysis proposed here will facilitate the detection of risk-taking behaviors and the development of effective strategies for their prevention.

Key words: Gender, risk-taking behaviors, health, interbehavioral psychology, youth.

\section{Introducción}

En años recientes, se puede observar un auge de los estudios con perspectiva de género (e.g. Montoya \& Harold, 2009; Oksuzyan et al. 2010; Phillips, 2005) abordándose el fenómeno desde distintas perspectivas teóricas (Martínez, 2010) y áreas disciplinares (Ghazal \& Gorman, 2011; Miño-Worobiej, 2008). Sin embargo, este auge y abordaje multidisciplinar no siempre ha traído consigo una mayor precisión y sistematización pues, en diversos momentos, se han mezclado conceptos, constructos y lógicas de las distintas disciplinas interesadas en el área, cayendo en lo que Ryle (1949/2005) ha dado en Ilamar errores categoriales. En este sentido, el presente artículo pretende plantear el fenómeno del género bajo criterios especificados por un particular punto de vista dentro de una disciplina particular, la psicología interconductual (e.g. Kantor, 1924; Ribes \& López, 1985; Ribes, 2007).

Algunos antecedentes del concepto de género En 1949 en su libro El Segundo Sexo, Simón de Beauvoir escribía "...no se nace mujer: se llega a serlo" ( $p .87)$, frase que, de manera informal, inaugura los estudios de género, pues plantea que las características humanas consideradas como "femeninas", son adquiridas por las mujeres mediante un complejo proceso de aprendizaje individual y social, en vez de derivarse "naturalmente" de su sexo (Butler, 1990/1996).

Posteriormente, se comienza a hablar de género con la intención de establecer la dife- rencia entre aquellas características fundadas en lo biológico (sexo) y aquellas relacionadas con las conductas atribuidas (por convención y práctica social) a los varones y las mujeres (Burin, \& Meler, 2000; Money, Hampson, \& Hampson, 1955; Stoller, 1968).

El feminismo académico anglosajón (e.g., Scott, 1986/1996) impulsó la categoría de gender (género) para tratar de subrayar la seriedad académica de los estudios relacionados con estas prácticas culturales diferenciales, debido a que dicho término suena más neutral y objetivo que el término "mujeres". De acuerdo con Lamas (1996), el término "Género" se ajusta de mejor forma a una terminología científica de las ciencias sociales desmarcándose, así, de las posturas políticas del feminismo.

Si bien de manera general parece existir un consenso respecto de la relación entre género y estilos de vida, siguen existiendo una diversidad de nociones y definiciones conceptuales que complican la conmensurabilidad de los diversos hallazgos empíricos obtenidos hasta el momento y, con ello, la comprensión del fenómeno que designan (Fernández, 1996; Palazzani, 2013).

Las hay desde las más sintéticas, como la propuesta por Butler (1990/1996) que sugiere que "género es (...) un estilo activo de vivir el propio cuerpo en el mundo" (p.308), hasta otras más elaboradas donde se identifica el sistema "sexo-género" como "...el conjunto de dispo- 
siciones por el que una sociedad transforma la sexualidad biológica en productos de la actividad humana, y en el cual se satisfacen esas necesidades humanas transformadas" (Rubin, 1975/1996, p. 37). Existen, también, definiciones que descansan en posiciones disciplinares más ligadas a lo que tradicionalmente se ha descrito como perteneciente al ámbito de lo psicológico, como lo propuesto por Burin y Meler (2000) que identifica al género como "... la red de creencias, rasgos de personalidad, actitudes, valores, conductas y actividades que diferencian a mujeres y a hombres" (p. 23).

Sin embargo, es dentro del ámbito disciplinar ${ }^{1}$ de lo social, donde se encuentra mayor cantidad de propuestas, como la de Scott (1986/1996) quien sugiere que el género se trata de "...un elemento constitutivo de las relaciones sociales basadas en las diferencias que distinguen los sexos (...) y es una forma primaria de relaciones significantes de poder (...) el género comprende cuatro elementos interrelacionados: a) Símbolos y mitos (...) b) los conceptos normativos (...) c) las nociones políticas, instituciones y organizaciones (...y...) d) la identidad subjetiva..." (p. 289).

O bien, la noción dada por West y Zimmerman (1999) en que se propone que el "...género es la actividad consistente en manejar una conducta determinada a la luz de conceptos normativos de actitudes y actividades apropiadas para la categoría sexual de cada persona (...) hacer género es emprendido por mujeres y hombres, cuya competencia como miembros de la sociedad es rehén de su producción. Hacer género implica un complejo de actividades perceptivas, interactivas y micro políticas socialmente guiadas que conforman actividades particulares como expresiones de la naturaleza femenina y de la masculina" (p. 112). Incluso, Connell (1995) sugiere que el género "... es una de las formas en las que se ordena la práctica social. En los procesos de género, la conducta cotidiana se

1 Para considerar una definición dentro de un determinado ámbito disciplinar (i.e., sociología, psicología), se tomó en cuenta el lugar dónde el autor pone el acento al momento de redactar su definición: bien en las propiedades del individuo; bien en las del colectivo social. organiza en relación con un ámbito reproductivo, definido por las estructuras corporales y los procesos de reproducción humana. Este ámbito incluye la excitación y el intercambio sexual, el nacimiento y cuidado infantil, las diferencias y semejanzas sexuales corporales (...) el género se organiza en prácticas simbólicas que pueden abarcar mucho más que la vida de un individuo" (p. 109).

En ese sentido, aunque el género se encuentra relacionado con el sexo biológico, éste no es el factor determinante ya que, de ser así, las mujeres (sexo) siempre tendrían las características comportamentales consideradas femeninas (género) y los varones las masculinas, por lo que serían universales (Lamas, 1996).

Existen algunos datos empíricos que apoyan la idea de que los rasgos/actitudes/conductas atribuidas a uno u otro género son relativamente estables (i.e., estereotipos) dentro de ciertos ámbitos culturales relacionados, posiblemente, con ciertos mecanismos de producción, distribución e intercambio de la riqueza, base de lo que, entre otros autores Ribes (1992), delimita el ámbito de lo social. Por ejemplo, Williams y Best (1990), en un estudio realizado en 27 países $^{2}$, en relación a la identificación de adjetivos que socialmente se relacionaban con el género, encontraron que existe un gran consenso transcultural en el contenido de los estereotipos de género. Así, a la masculinidad le fueron atribuidos rasgos instrumentales y de agente tales como agresividad, valentía, competitividad, fuerza, poder e independencia; mientras que a lo femenino se le atribuyeron rasgos expresivos y de comunidad tales como delicadeza, dependencia, sumisión, fragilidad, preocupación por los demás, entre otros.

En síntesis, a partir de lo descrito hasta ahora, se puede afirmar que el género: a) es una construcción social (convencional), por lo que cada sociedad construye sus propias normas de género; b) es algo aprendido; c) se sanciona a los individuos su adhesión, o no, a dichos cons-

2 Los investigadores estudiaron las respuestas de 300 participantes de 27 países tan disimiles como Brasil, Canadá, Nigeria, Pakistán, Estados Unidos de América, Finlandia e India, entre otros. 
tructos; d) es susceptible de ser modificado; y e) aunque se basa en las diferencias biológicas (sexo), no es algo intrínseco a ello.

Apuntes sobre la postura interconductal ${ }^{3}$ Con el fin de abonar al entendimiento multidisciplinar de la noción de género, y partiendo del supuesto de que la multidisciplinariedad sólo es posible a partir de la diferenciación inicial de campos y objetos de estudios claramente delimitados (Ribes, 2010), nuestro interés, a diferencia de otras disciplinas que abordan dicho fenómeno desde una perspectiva social (i.e., sociología, antropología, incluso algunas aproximaciones psicológicas), es analizarlo desde un punto de vista específicamente psicológico, es decir, identificar los factores que intervienen para que se establezca el ajuste individual, en situaciones particulares, dentro de una determinada práctica social.

Bajo una lógica de campo, de acuerdo con Kantor (1924), Ribes y López (1985) y Ribes (2007), el comportamiento del individuo no puede entenderse (y por tanto, explicarse) independientemente de la circunstancia en que se presenta. Dicha circunstancia, que para fines analíticos es considerada como una situación, supone una red de relaciones de contingencia ${ }^{4}$ entre los ele-

3 Cabe mencionar que, debido a los alcances e intereses del presente escrito, la descripción de la postura interconductual será sucinta y, con ello, necesariamente incompleta. Se invita al lector interesado en ahondar en los principios e implicaciones de esta postura, revisar los textos de Kantor (1924, 1963/1990), Ribes y López (1985); Ribes, (2007) y Ribes, Rangel y López (2008).

4 Si bien el concepto de contingencia tiene diferentes acepciones, no sólo en disciplinas distintas como la física o las del área social, sino particularmente dentro de la psicología catalogada como conductista, el uso que se le da en el presente escrito es consistente con lo propuesto por, entre otros, Ribes \& López (1985). En este sentido, entenderemos contingencia como relación de dependencia entre elementos que participan en el campo psicológico, relaciones cuyo establecimiento suponen la estructuración de una función y que componen el entramado del campo interconductual. mentos que componen el segmento de análisis, que incluyen, entre otros: objetos, eventos y personas (incluido el individuo focal de análisis). Una característica que es necesario resaltar es que el criterio de pertenencia a la situación no se encuentra, necesariamente, determinado por criterios de contigüidad temporal o espacial, sino por criterios funcionales en términos del establecimiento de relaciones de contingencia. De igual forma, se asume que el individuo siempre, y en todo momento, se está comportando, es decir ajustándose a los cambios que el ambiente "propone" no sólo a partir de la afectación que el mismo comportamiento del individuo provoca sino, también, por la acción y efectos de otros individuos y factores incluidos en el campo interconductual.

Entre los elementos generales que, se considera, pertenecen al Ilamado campo interconductual se encuentran: a) objetos y eventos de estímulo con los que hace contacto el individuo, b) variables organísmicas relacionadas con los sistemas reactivos con que cuenta el individuo en cuestión (que permiten entrar en contacto con los objetos y eventos de estímulo), c) factores situacionales que pueden alterar las relaciones que se actualizan (i.e., afectos, motivaciones) y d) factores disposicionales que probabilizan ciertos contactos presentes. Cada uno de estos elementos puede adquirir un valor particular, lo que determinará la circunstancia en que sucede el comportamiento.

Así, la configuración particular que adquiere un campo psicológico específico, que establece la diferencia respecto de otros campos o situaciones, depende no sólo de los valores de cada uno de los elementos que lo componen, sino también de las relaciones que se establecen entre ellos. A su vez, las relaciones de dependencia que se establecen, se encuentran probabilizadas por una serie de elementos que cumplen con una función disposicional ${ }^{5}$ : a) la historia interactiva del individuo, que implica las formas peculiares de interacción que ha desarrollado a lo largo de

5 Hablar de una función disposicional es hablar de la alteración de la probabilidad de ocurrencia de eventos y/o relaciones de contingencia en el campo psicológico.

vol. 6 num. 2 NOV-2014/APR-2015 
su ontogenia, y b) aspectos relacionados con el ámbito de lo social (i.e., instituciones, normatividad explícita e implícita, sistemas de valores, creencias).

Asumir el comportamiento como parte de un campo psicológico implica, como una de sus consecuencias lógicas, la imposibilidad de hablar en términos causales lineales entre eventos del ambiente y reacciones del organismo; es decir, si bien se asume que el ambiente, segmentado con fines analíticos en términos de objetos de estímulo, relaciones de contingencia, factores situacionales, factores disposicionales, entre otros, posibilita ajustes particulares del individuo a partir de su comportamiento, no es posible siquiera sugerir que un evento particular del ambiente produjo un comportamiento particular; no es posible hacerlo porque, como ya se ha afirmado, el comportamiento es visto como una función de la estructuración del campo psicológico, estructuración que está determinada por todos los elementos que lo componen y las relaciones que se establecen entre dichos elementos, incluidos aspectos relacionados con el individuo mismo.

A pesar que desde este enfoque de campo no se deben enunciar declaraciones lineales de causalidad, sí es factible hacer un análisis de los factores que probabilizan la configuración de un segmento conductual de una determinada manera. A continuación se propone una forma en que se puede analizar el fenómeno del género.

\section{El género desde una perspectiva interconductual}

Siguiendo la lógica interconductual previamente descrita, el análisis psicológico de la noción de género requiere, necesariamente, la identificación de dos dimensiones: una social o cultural y la otra individual o conductual. Ambas dimensiones son pertinentes para la comprensión del género como factor que delimita la pertinencia del comportamiento en una situación determinada con base en criterios socialmente convenidos.

La dimensión social del género corresponde a los criterios, convencionalmente establecidos, que un determinado grupo social (i.e. familia, cultura, instituciones) establece como marco regulador respecto de las prácticas, usos y costumbres de sus miembros para permitir la convivencia. Esto se ve reflejado en sus instituciones y/o costumbres, las normas formales e informales que prescriben, a través de las cuáles se evalúa y se sanciona el ajuste del individuo de acuerdo, por ejemplo, con su sexo. Todos estos aspectos tienen que ver con lo que se ha denominado cultura, entendida como: a) el conjunto de prácticas mediante las que se expresan las convenciones $y, b$ ) el marco de referencia en el que se adquiere y ejercita el comportamiento de los individuos (Ribes, 1990). En este sentido, las conductas relacionadas con el género, son conductas valoradas a partir de un criterio socialmente establecido.

Desde este particular punto de vista psicológico, se asume que la dimensión social o cultural puede analizarse identificando los factores macrocontingenciales de un segmento de interacción. Dichos factores tienen que ver con el sistema de valores (i.e. normas formal e informalmente instituidas, ejemplares de conducta, principios) que sirven como criterio de evaluación de la conducta de los individuos. No obstante, de acuerdo con Ribes (1990) los valores no son entidades que regulan el comportamiento del individuo desde el exterior, sino que son una parte de las prácticas conductuales que regulan la transmisión, reproducción y ejercicio de formas particulares de comportamiento que afectan a grupos de individuos de acuerdo, en el caso del género, con lo que en una determinada cultura se ha sancionado como pertinente para los miembros de cada sexo.

Ribes desarrolló una propuesta para sistematizar el análisis del comportamiento en situación, el cual denominó análisis contingencial (1990). En este caso para identificar la dimensión psicológica del género, es necesario, identificar las macrocontingencias: 1) las prácticas sociales dominantes relativas al género (i.e., lo que deben hacer las personas que pertenecen a un sexo o a otro); 2) las prácticas lingüísticas que referencian efectos reguladores por parte del grupo social normativo (i.e., lo que se dice acerca de las consecuencias establecidas respecto de lo que se debe o no debe hacer), 3) los indi- 
viduos y grupos de referencia vinculados con las conductas y con las prácticas lingüísticas de referencia (i.e., quién hace, quién dice lo que se debe hacer y quién sanciona); 4) correspondencia lingüística del individuo con otros grupos (i.e., si suscribe o no, lo que su(s) grupo(s) de referencia afirman); 5) correspondencia funcional entre prácticas sociales e individuales (i.e., si ajusta su comportamiento a lo esperado por otros); 6) comparación entre los efectos microcontingenciales y la normatividad (i.e., si al ajustarse a los criterios del grupo, obtiene los resultados esperados).

El propio Ribes sugiere que los factores macrocontingenciales forman parte de un grupo o sistema social al que el individuo se integra y trascienden la dimensión individual, en el sentido de que conforman un sistema establecido al que el individuo se integra, y que es susceptible de modificar, a partir de su práctica. Dichas prácticas sociales son criterios de pertinencia del actuar en situación y delimitan el dominio o contenido que enmarca, o dan sentido, a las prácticas individuales. Existen prácticas específicamente relacionadas con el género, las cuales se pueden articular de distintas maneras: a) en forma de indicaciones, advertencias, comparaciones o ejemplares de tipo general que prescriben el "deber ser" dependiente del sexo de referencia o, b) como "deber hacer" particulares derivados de dichos ejemplares, regulando la situación por medio de sanciones. Esta prescripción, modulación, y sanción diferenciada para cada sexo articula la noción de género.

De esta manera, las normas sociales relacionadas con el género no constituyen un conjunto supraordenado o externo a la propia práctica individual, sino un conjunto de creencias con las que el individuo entra en contacto a través de la práctica interrelacionada con los demás individuos de su(s) grupo(s) de pertenencia. Dicho contacto no ocurre como algo artificial o forzado, sino que forma parte del conjunto de prácticas cotidianas del individuo.

Una segunda dimensión al analizar el género, desde esta perspectiva, tiene que ver con las prácticas individuales que son prescritas, evaluadas y sancionadas de acuerdo con las normas socialmente establecidas. Si bien las prácticas de género están delimitadas socialmente y no pueden comprenderse al margen de su dimensión social, el ajuste al conjunto de normas y criterios culturales que delimitan el género es individual. El individuo puede ajustar o no su conducta a las prácticas convencionales relacionadas con el género. Los usos y los criterios se actualizan a través de la propia conducta del individuo en el contexto de las normas implícitas y explícitas que culturalmente regulan las prácticas de género. La conducta individual se valora como ajustada o desajustada en relación con dichas prácticas normadas.

Este segundo grupo de factores relacionado con el comportamiento en situación, puede describirse de acuerdo con distintos tipos de relaciones microcontingenciales: 1 ) Conductas instrumentales focales (i.e., forma en que se presenta el comportamiento analizado); 2) condiciones situacionales, que poseen funciones disposicionales (en tanto colecciones de ocurrencias que condicionan probabilísticamente una relación: capacidades, inclinaciones, propensiones y tendencias); 3) personas implicadas funcionalmente en la situación (i.e., mediador: determina el tipo de interacción; mediado: aquel que es regulado por el mediador); 4) efectos contingenciales (i.e., consecuencias de la interacción: directas, indirectas, para sí mismo o para otro(s)). En la Tabla 1 se enumeran los elementos micro y macrocontingenciales pertinentes al análisis de la noción de género. 
Tabla 1. Elementos propuestos para analizar el ajuste individual en situación

Identificación de la situación a analizar

\begin{tabular}{|c|c|}
\hline Factores Microcontingenciales & Factores Macrocontingenciales \\
\hline 1. Conductas instrumentales focales & 1. Prácticas sociales dominantes \\
\hline $\begin{array}{l}\text { 2. Condiciones situacionales, que poseen funciones } \\
\text { disposicionales (capacidades personales, } \\
\text { propensiones, tendencias) }\end{array}$ & $\begin{array}{l}\text { 2. Prácticas lingüísticas que referencian afectos } \\
\text { reguladores } \\
\text { 3. Individuos o grupos de referencia }\end{array}$ \\
\hline $\begin{array}{l}\text { 3. Personas implicadas funcionalmente en la } \\
\text { situación (mediador y mediado de la situación, } \\
\text { otros) } \\
\text { 4. Efectos contingenciales }\end{array}$ & $\begin{array}{l}\text { 4. Correspondencia lingüística del individuo con } \\
\text { otros grupos } \\
\text { 5. Correspondencia funcional entre prácticas sociales } \\
\text { e individuales } \\
\text { 6. Comparación entre los efectos } \\
\text { microcontingenciales y la normatividad }\end{array}$ \\
\hline
\end{tabular}

Nota: Representación sintetizada de la propuesta de análisis contingencial de Ribes (1990, pp. 120-123).

Así pues, el género, como práctica social reconocida, implica identificar los criterios sociales que delimitan la conducta pertinente en relación con el sexo y la correspondencia o falta de ella entre esos criterios y el propio comportamiento. Solo es posible predicar la adscripción a un género cuando el individuo puede reconocer los criterios bajo los cuales su actuar es pertinente, en caso contrario se dirá que se ajusta por imitación u obediencia.

En la Figura 1 se esquematiza la forma en que se puede establecer el ajuste individual a un determinado ejemplar de género. Se evoca lo planteado por Connell (1995), en el sentido de que en cada sociedad existen cuando menos tres formas de configurar el género (hegemónico, alternativo y estigmatizado). En la parte superior del esquema se ejemplifican tres de las distintas configuraciones en que se puede estructurar el género en la sociedad occidental contemporánea. En nuestras sociedades es relativamente fácil encontrar la convivencia de estas tres configuraciones: familia tradicional, donde los roles de género y la división sexual del trabajo están claramente definidos; una familia o pareja alternativa, en donde los roles de género se siguen pero con ciertas concesiones; y relaciones o grupos LGBT (i.e., Lesbico, Gay, Bisexual y Trans), en donde los roles de género rompen drásticamente con lo socialmente esperado.
Cuando un individuo se enfrenta a la disyuntiva de ajustar su comportamiento a un determinado ejemplar de género, lo hace en función, en primer lugar, de los distintos modelos que tiene a su alcance, así como de los factores disposicionales, tomando mayor relevancia las personas que funcionalmente se encuentren en la interacción, y las posibles consecuencias que tenga el comportamiento del individuo.

$$
\begin{gathered}
\text { El género y su relación con conductas de } \\
\text { riesgo para la salud }
\end{gathered}
$$

En México, como en muchos otros países del mundo, se ha sugerido la existencia de un sesgo significativo entre algunos indicadores de riesgo entre hombres y mujeres, sobre todo entre jóvenes. Por ejemplo, de acuerdo con datos de la encuesta nacional realizada por el INEGI durante el año 2010 el número total de muertes en jóvenes de entre 15 y 29 años ascendió a 37,519, de los cuales $76.05 \%$ fueron hombres y el $23.95 \%$ mujeres (2012). En un análisis más detallado del motivo de dichos fallecimientos, se encuentra que en el $70.4 \%$ de los hombres fue por causas violentas (i.e., accidentes, homicidios), mientras que en mujeres representó el $36.4 \%$.

Este fenómeno se repite en estadísticas de delincuencia donde, por ejemplo, la cantidad de personas sentenciadas en el fuero común en el 2010 ascendió a 120,177 (INEGI, 2011), de los 
cuales $90.7 \%$ fueron hombres y $9.2 \%$ mujeres, lo cual puede sugerir que el factor género puede estar relacionado con ese tipo de conductas de riesgo.

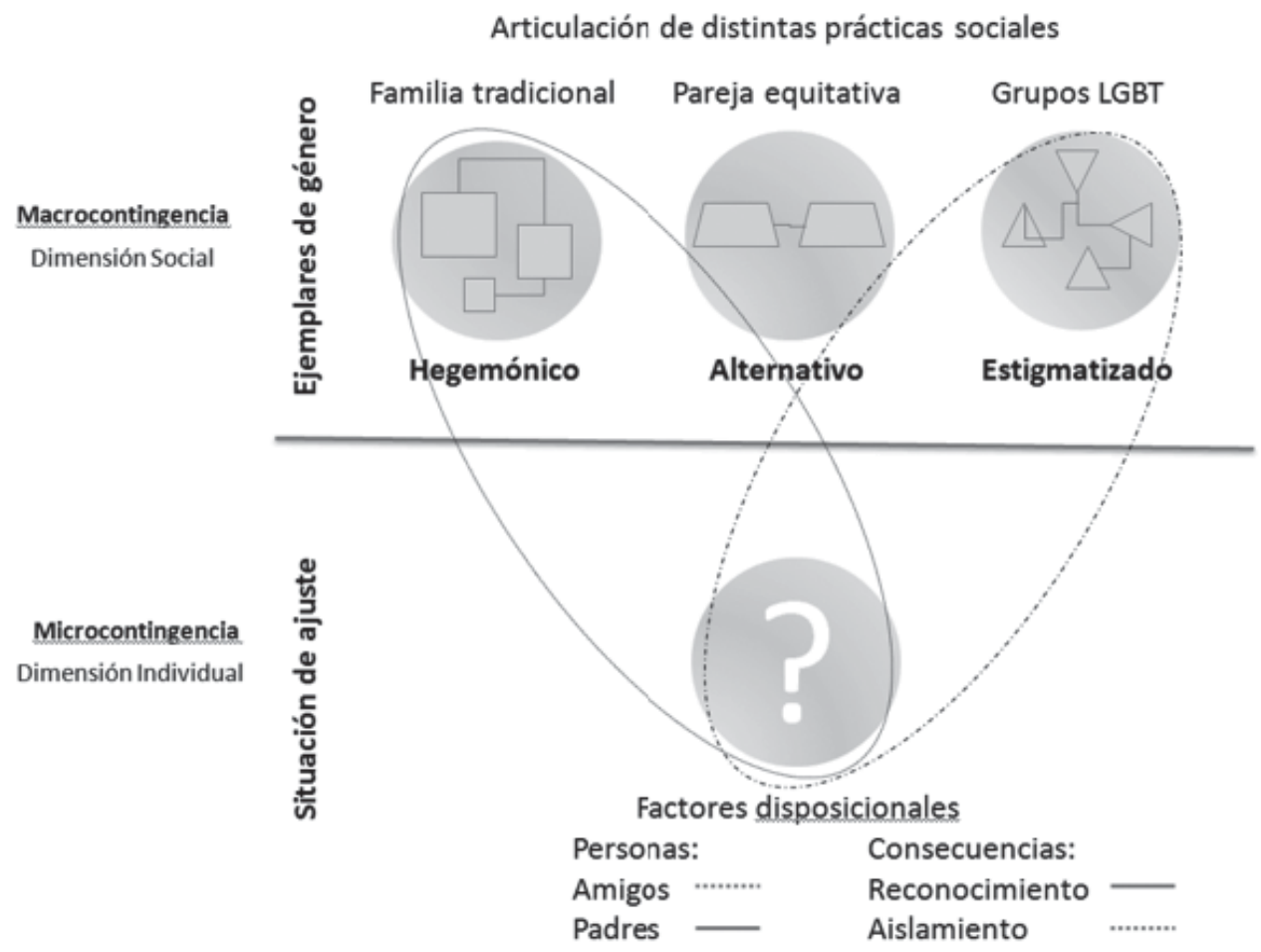

Figura 1. Esquematización del ajuste individual a un determinado ejemplar de género

A partir de lo que se ha dicho hasta el momento, se puede señalar que el género implica a las prácticas sociales relacionadas con la forma en que se debe reaccionar (deber hacer y decir) en situaciones generales. Estas maneras, que suponen la normatividad social respecto de cómo comportarnos dependiendo del sexo biológico de pertenencia, se actualizan en las prácticas individuales (instrumentales y referenciales) que se ejercen en situaciones particulares, prácticas que permiten el ajuste a los criterios convencionales impuestos.

Tales prácticas individuales, se realizan y adquieren a partir de lo experimentado en la historia personal; se aprende a hacer y a decir en situación, se aprende a decir como justificación del actuar en situación. Este hacer y decir se realiza en relación con lo hecho y dicho por otros significativos (ejemplares) que se encuentra, a su vez, en congruencia con lo que se ha convenido (en términos de una práctica consistente) que debe o no hacerse y decirse, y de las consecuencia de hacerlo o no de una forma determinada.

El riesgo y la prevención son maneras que permiten cualificar las interacciones particulares y las tendencias interactivas en relación con el acercamiento o alejamiento de fuentes potenciales de daño (e.g., Ribes, 1990). Si, como se dijo, se asume el aprendizaje de reacciones ante situaciones a partir del seguimiento de modelos (ejemplares), y que las tendencias suponen la actualización consistente de dichas reacciones en situaciones funcionalmente semejantes, parece evidente que las prácticas individuales están teñidas del deber hacer, en tanto que se aprende a hacer de forma consistente de la manera en que otros suelen hacerlo dado que de esa manera "debe" hacerse, a partir de las consecuencias del propio comportamiento. Es decir, se aprende a hacer lo que se debe, en la medida en que se 
ve y escucha cómo se deben hacer las cosas, y de las consecuencias obtenidas por hacerlo de esa forma, directa o indirectamente.

En este sentido, se aprende a reaccionar de una manera que puede calificarse como arriesgada o preventiva, porque como individuo que pertenece a un cierto grupo, se ha visto que así se ha hecho, dado que así debe hacerse, y las consecuencias obtenidas por el hacer, o no, en situaciones particulares.

El hecho de que haya más hombres que mujeres en determinadas situaciones de riesgo (i.e., lesiones, fracturas), y más mujeres que hombres en otras (i.e., violaciones), parece indicar que las prácticas sociales relativas al género (ejemplares) suelen ser sesgadas; en el caso de ellos, preponderantemente orientadas a la instrumentalización del riesgo como algo activo, propositivo (i.e., conducta como elemento mediador, que estructura el campo); mientras que en ellas, el riesgo suele darse más en un sentido pasivo, donde la situación se estructura como ocurrencia de eventos, por lo que suele analizarse este comportamiento como ajeno al segmento conductual de riesgo (i.e., conducta como elemento auspiciador de la situación). Cabe aclarar que lo instrumental o pasivo de las conductas, no son privativos de un determinado género, sin embargo, históricamente a raíz de la división social del trabajo, convencionalmente al hombre se le asigna el rol de proveedor y garante de la seguridad de su grupo social (familia); mientras que de ellas se espera el rol complementario, que se encarguen de atender y criar a los hijos, conformándose con las capacidades del hombre de la casa para sacar a su familia adelante (e.g., Roca, 2011).

Desde una perspectiva de campo interconductual, hacer o dejar de hacer cosas, dadas ciertas circunstancias, independientemente de que no sean el elemento crítico para la estructuración del segmento conductual, también es un componente que forma parte de la estructura contingencial del campo, lo que lo hace indispensable para el análisis de la configuración de las situaciones calificadas como de riesgo.

En este sentido es importante identificar tanto las conductas instrumentales de riesgo (i.e., consumo de drogas, participación en riñas), como aquellas conductas que auspician tales situaciones riesgosas (i.e., no responder a señales que anticipan el evento, aceptar condiciones de inequidad) como elementos constituyentes del segmento de análisis. Por lo general, las estadísticas de riesgo hacen referencia, y orientan su análisis a aspectos de instrumentalidad (i.e., conductas activas de riesgo); mientras que, a las conductas auspiciadoras no se les considera como parte de un segmento de riesgo sino que se habla de ellas, como una ocurrencia ajena al individuo.

Así, el comportamiento del individuo en situaciones particulares, se ajusta a criterios convenidos socialmente, entre los que se encuentran aquellos relacionados con el género. Por ello, en la mayoría de las interacciones humanas, se pueden identificar conductas en las que prevalecen criterios derivados de uno o varios ejemplares de género, que pueden modular el acercamiento o alejamiento de fuentes potenciales de riesgo. Como se ha mencionado, el análisis del segmento conductual en donde se identifica la actualización de ejemplares de género a través de haceres y decires específicos, requiere la identificación de los elementos que componen dicho segmento, es decir, factores micro y macrocontingenciales.

\section{Ejemplificación del análisis propuesto} A manera de ejemplo, se analiza una situación que un joven relató ${ }^{6}$, en donde el primer elementos está conformado por la situación en sí misma: se trata de la interacción entre un hombre $(\mathrm{H})$ de 20 años de edad, estudiante de $6^{\circ}$ semestre de preparatoria, y una mujer $(M)$ a quien tiene poco de conocer y con quien $\mathrm{H}$ quería tener relaciones sexuales. $\mathrm{H}$ afirma: "ni la quería ni nada.... yo quería llevármela acá a la cama pero ella decía que no, porque no éramos novios y pues le tuve que decir". Él le pidió que fueran novios y momentos después ya estaban teniendo relaciones sexuales.

En relación con el segundo componente, analizar los elementos que poseen funciones disposicionales: $\mathrm{H}$ ha aprendido que para obtener satisfactores de otras personas, debe identificar

6 Solo de forma ilustrativa, se toma el testimonio de un participante en una investigación empírica desarrollada por este equipo de trabajo. 
qué es lo que los otros necesitan para acceder (i.e., intercambio de favores, negociación, coerción). Mientras que $M$ ha aprendido a ceder bajo ciertas circunstancias (i.e., declaración verbal de compromiso). Dado que estos aprendizajes se actualizan ${ }^{7}$ en distintos episodios, pueden considerarse como inclinaciones individuales que juegan un papel disposicional, es decir, en determinadas circunstancias probabilizan ciertos comportamientos. En este ejemplo, para que $\mathrm{H}$ logre su objetivo (tener relaciones sexuales); debe identificar lo que $M$ "necesita" (i.e., "compromiso", "amor"), al tiempo que a $\mathrm{H}$ no le interesa comprometerse. El hecho de que $\mathrm{H}$ llevara más de dos años sin tener novia al momento de la entrevista, podría ser un indicador de la disposición que tiene $\mathrm{H}$ a no comprometerse, además prefiere entrar en contacto con mujeres que considera más inocentes, por lo que suele ir a pueblos para lograr sus objetivos. Mientras que $M$ sólo accede a tener relaciones sexuales bajo condiciones de "compromiso y afecto" (i.e., noviazgo y amor).

El tercer factor del análisis implica identificar al elemento que funge como mediador de la interacción; en el presente caso, $\mathrm{H}$ es quien propicia las condiciones necesarias para que el segmento conductual se articule de la forma como él lo desea (i.e., tener relaciones sexuales con $M$ ). Un punto medular que permite identificarlo como el mediador de la interacción es cuando le dice lo que ella "necesita" escuchar, a pesar de no querer ese tipo de compromisos.

Finalmente, el cuarto elemento microcontingencial es el relacionado con el análisis de los efectos implicados en dicha contingencia; se puede identificar que cuando $\mathrm{H}$ le declara su amor a $M$, consigue tener relaciones sexuales (efecto directo para él), adquiriendo (aunque sin asumir), de manera indirecta, un "compromiso" no deseado (tener novia). Su acciones tienen, de igual forma un efecto sobre los otros, pues $M$ hizo algo que probablemente no hubiera hecho, si $\mathrm{H}$ no hubiese actuado como lo hizo.

7 Actualización es utilizada en términos rayleanos, en el sentido de que una disposición se actualiza en un acto concreto. La fragilidad del cristal se actualiza cuando se rompe.
En cuanto al análisis de los factores macrocontingenciales: el primer grupo hace referencia a la identificación de las prácticas sociales dominantes. En este caso, uno de los mandatos de la masculinidad hegemónica es: "Los hombres que tienen más experiencia sexual son más hombres", esto es, para Ser muy hombre, tienes que tener (Hacer) mucha práctica erótica heterosexual. En contraposición, con los mandatos de la feminidad hegemónica, que afirman: "una mujer decente sólo debe entregarse cuando hay un compromiso de por medio", en este caso el deber Ser de la mujer pasa por mantenerse lo más pura y casta que le sea posible, para lo cual su Hacer consistirá en rechazar aquellas situaciones que impliquen erotismo.

Dentro del segundo grupo de factores se encuentra la identificación de las prácticas lingüísticas que referencian efectos reguladores sobre el comportamiento. Entre los hombres es común escuchar sentencias como: "si no tienes relaciones sexuales con mujeres van a pensar que eres joto, y vas a perder el respeto"; mientras que para ellas las prácticas lingüísticas van en el sentido de: "si tienes muchas relaciones sexuales te van tachar de 'zorra' y los hombres no te van a tomar en serio".

Un tercer componente hace referencia a la identificación de los individuos, o grupos de referencia, vinculados a prácticas sociales que regulan la conducta (microcontingencias ejemplares). En general, se pueden identificar, cuando menos, dos microcontingencias ejemplares estrechamente relacionadas con el episodio analizado: a) una cuando $\mathrm{H}$ refiere que su papá "era muy cabrón", "metía viejas a su casa y le valía"; y b) la segunda al comentar de sus tíos: "Se van dos o tres meses a Estados Unidos y viven un año aquí, de mantenidos y las esposas trabajando, y ellos mujeriegos y tomando". En ese sentido, $\mathrm{H}$ se ajusta con base en los modelos que le brinda su entorno inmediato significativo, pues es donde ha aprendido la forma de vivir, y justificar, su actuar como hombre (i.e., masculinidad).

La evaluación e identificación de la correspondencia sustitutiva del individuo con diversos grupos relativos a diversas prácticas, es decir, la 
correspondencia entre lo que el individuo analizado dice de su hacer y las prácticas lingüísticas de su(s) grupo(s) de referencia, integran un cuarto grupo de factores. Además de lo referido sobre el papá y los tíos, H comenta que, para demostrar su masculinidad, los amigos argumentan cosas tales como: "Los hombres a veces tienen que echarle mentiras a sus amigos como 'Ayer agarré una vieja', para que no te anden diciendo que eres joto o algo así".

Comparar la(s) conducta(s) del individuo con las prácticas sociales que la contextualizan y regulan, es la quinta etapa del análisis; en este sentido, se pueden identificar dos mandatos que claramente se actualizan en el comportamiento de $\mathrm{H}: 1$ ) a pregunta expresa de cómo debe actuar un hombre, él responde: "con carácter fuerte, debe de mandar todo a la fregada, que le valgan madre las cosas", y esto se ve actualizado cuando se le pregunta si él actúa de esa manera y responde "A veces tengo que estar urgente en la escuela, y no voy. No me gusta traer muchas cosas en la cabeza, prefiero mandar todo por un lado, concentrarme nada más en lo principal". Y, 2) la demostración de la potencia sexual, haciéndolo o diciéndolo, la cual ha sido expuesta en la narración previa.

Los ejemplares a los que $\mathrm{H}$ se ha adherido, en cuanto al ejercicio de su sexualidad (i.e. parejas ocasionales, múltiples compañeras, falta de compromiso) y su comportamiento en general (i.e. poco tacto en sus interacciones, irresponsabilidad), ha propiciado que $\mathrm{H}$ priorice ciertas actitudes y comportamientos que aumentan la probabilidad de que se enfrente a situaciones potenciales de riesgo, que implican tanto el daño directo (i.e. contraer enfermedades de transmisión sexual, riñas por celos de otros hombres), como la pérdida de oportunidades de desarrollo personal (i.e., no ir a la escuela, ni asumir otro tipo de compromisos).

Algo semejante puede argumentarse para $M$, quien al adherirse a un determinado ejemplar de género (i.e. mujer tradicional, sumisa, recatada), aumenta la probabilidad de que su comportamiento la sitúe en una posición tal que si H (o cualquier otro agente) actúa, la situación implicará mayor riesgo para $M$. Por ejemplo, el hecho de que $M$ asuma que la declaración de amor de $\mathrm{H}$, como muestra de compromiso, debe ser recompensada, en un sentido de trueque, con una muestra de amor y compromiso equivalente, en este caso, las relaciones sexuales, esto es aprovechado por $\mathrm{H}$ para lograr sus objetivos, poniéndola a ella en una posible situación de vulnerabilidad.

Parece claro que no sólo el hacer de $\mathrm{H}$ es relevante para la estructuración del segmento conductual, sino que el hacer de $M$ también forma parte del mismo. Por lo tanto, no se podría afirmar que $M$ es una víctima, a la que le ocurren las cosas, sino que también es corresponsable de la forma en que dicho segmento conductual se articula, pues su hacer o no hacer posibilita cierto tipos de contacto y de establecimiento de relaciones de dependencia entre los elementos que componen el campo interconductual. Como se puede observar, una de las virtudes de este modelo es que nos permite trabajar con los distintos elementos que participan en la interacción, así como con las relaciones que se establecen entre ellos, esto aumenta la probabilidad de generar estrategias preventivas exitosas, ya que permite identificar con precisión los elementos que interactúan y su rol en la estructuración del campo, así como diversificar alternativas de solución, ya sea trabajando con el agente activo de la interacción (mediador), como con el o los agentes receptivos de la misma (mediados), así como con eventos que pueden estar participando como factores disposicionales en la interacción.

\section{A manera de conclusiones}

A lo largo del artículo se ha insistido en la necesidad de delimitar con claridad las distintas áreas del conocimiento que intervienen en el fenómeno del género para un abordaje interdisciplinario, que nos permita una mejor comprensión del tema. Para ello se ha tomado como ejemplo la relación del género y las conductas de riesgo para la salud, ya que desde hace varios años se ha venido acumulando evidencia que apoya una fuerte relación entre el ejemplar de género al que los y las jóvenes se adhieren y las conductas de riesgo que pueden llegar a asumir (Sabo, 2000; Montoya \& Harold, 2009). 
Se considera que una buena estrategia para tal propósito es la delimitación de los distintos ámbitos en los que se puede hablar del factor de género. En ese sentido se plantea que el análisis del fenómeno se debe establecer en varios niveles: a) los que estarían constituidos por lo que desde la perspectiva interconductual se denominan macrocontingencias, punto de interés de las distintas disciplinas sociales, la psicología interconductual contempla estos factores para el análisis de la situación en donde se desarrolla el individuo, pero no pretende explicarlos; b) el análisis del ajuste individual y las relaciones de dependencia que estructuran el campo psicológico del individuo (microcontingencias), son aspectos netamente psicológicos, y por ende, debería ser la psicología quien dé cuenta de ellos.

Respecto del análisis funcional de las conductas de riesgo, se puede afirmar que la masculinidad hegemónica está relacionada con elementos preponderantemente activos de la conducta; mientras que el riesgo relacionado con las nociones de femineidad son más de tipo receptivo. En ese sentido, dado que las relaciones entre los elementos y los individuos que participan en un determinado segmento conductual se estructuran funcionalmente, esa "receptividad" (no actuación), en términos funcionales es una forma de comportarse y por ende susceptible de modificar cualitativamente la situación si se cambia la forma de responder ante ella. Es justo ahí donde los alcances de la propuesta tiene su máxima dimensión, ya que el hecho de que cada uno de los participantes en un segmento conductual dado, se responsabilice de sus haceres y decires, podría coadyuvar a que las mismas personas sean capaces de identificar situaciones potenciales de riesgos y hacer algo para prevenir y anticipar su ocurrencia.

Cabe aclarar que hablar de los distintos modelos o ejemplares de género no implica juzgarlos, en sentido de que uno u otro es mejor o peor que los demás, simplemente que el análisis funcional de cada uno de ellos implica un aumento en la probabilidad de enfrentarse a determinadas situaciones de riesgo, si se vive el género de una u otra manera.

\section{Bibliografía}

Beauvoir, S. (1949). El Segundo sexo. Madrid: Random House Mondadori.

Burin, M., \& Meler, I. (2000). Varones: Género y Subjetividad Masculina. Bueno Aires: Paidos.

Butler, J. (1990/1996). Variaciones sobre sexo y género: Beauvoir, Witting y Foucault. En L. Marta, El Género La contrucción de la diferencia sexual (pp. 303-326). México: Púrrua/pueg.

Connell, R. (1995). Masculinidades. México: UNAM.

Fernández, J. (1996). Sexo, Sexología y generología en varones y mujeres desarrollo de la doble realidad del sexo y del género. Madrid: Piramide.

Ghazal, R. J., \& Gorman, B. K. (2011). Gender and Health Revisited. En B. Pescosolido, J. Martin, J. McLeod, \& A. Rogers (eds.). Handbook of the Sociology of Health, Illness, and Healing (pp. 411-429). New York: Springer.

INEGI. (2011). Estadísticas Judiciales en materia penal de los Estados Unidos Mexicanos. Recuperado de http://www.inegi.org.mx/ prod_serv/contenidos/espanol/bvinegi/ productos/continuas/sociales/judiciales/2011/ judiciales_2010.pdf

INEGI. (2012). Instituto Nacional de Estadística y Geografía. Recuperado de http://www. inegi.org.mx/sistemas/olap/proyectos/bd/ continuas/mortalidad/mortalidadgeneral. asp? $\mathrm{s}=\mathrm{est} \& \mathrm{c}=11144 \&$ proy=mort_mg

Kantor, J. R. (1924). Principles of Pshychology. New York: Alfred Knop. doi:10.1037/10752-000

Kantor, J. R. (1963/1990). La evolución científica de la psicología. México, D.F.: Trillas.

Lamas, M. (1996). El género: La construcción cultural de la diferencia sexual. México: Purrúa.

Martínez, A. (2010). Identificación melancólica y constitución de la identidad de género masculina. Aportes del psicoanálisis a los estudios contemporáneos de género. Revista de Psicología, 19(2) 79-102.

Miño-Worobiej, A. (2008). Imágenes de género y conductas sexual y reproductiva. Salud Pública de México, 50(1), 17-31. doi:10.1590/ S0036-36342008000100008

Money, J., Hampson, J.G., \& Hampson, J.L. (1955). An examination of some basic sexual concepts: 
The evidence of human Hermaphroditism. Bolletin of the Johns Hopkins Hospital, 97(4), 301-319.

Montoya, E., \& Harol, J. (2009). La Articulación de las categorías de género y salud: un desafía inaplazable. Gerencia y Políticas de Salud, 9(17), 106-122.

Oksuzyan, A., Crimmins, E., Saito, Y., O'Rand, A., Vaupel, J., \& Christensen, K. (2010). Crossnational comparison of sex differences in health and mortality in Denmark, Japan and the US. European Journal of Epidemiology, 25(7), 471-480. doi: 10.1007/s10654-0109460-6.

Palazzani, L. (2013). Gender in Philosophy and Law. New York: Springer. doi:10.1007/978-94007-4991-7

Phillips, S. (2005). Defining and measuring gender: A social determinant of health whose time has come. International Journal for Equity in Health,11(4). doi:10.1186/1475-9276-4-11

Ribes, E. (1990). Psicología y Salud. México D.F. : Trillas.

Ribes, E. (1992). Factores Macro y Microsociales participantes en la regulación del comportamiento psicológico. Revista Mexicana de Análisis de la Conducta, 18, 39-55.

Ribes, E. (2007). Estados y límites del campo, medios de contacto y análisis molar del comportamiento: reflexiones teóricas. Acta Comportamentalia, 15(2), 229-259.

Ribes, E. (2010). Lenguaje ordinario y lenguaje técnico: Un proyecto de curriculo universitario para la Psicología. Revista Mexicana de Psicología , 27, 55-64.

Ribes, E., \& López, F. (1985). Teoría de la Conducta: Un análisis de campo y Paramétrico. México: Trillas.

Ribes, E., Rangel, N., \& López, F. (2008). Análisis teórico de las dimensiones funcionales del comportamiento social. Revista Mexicana de Psicología, 25 (1), 45-57.

Roca, C. N. (2011). Interacción grupal y violencia sexista: marco conceptual. En N. Roca y J. Masip (eds.). Intervención grupal en violencia sexista. (pp. 15-80). España: Herder.

Rubin, G. (1975/1996). El tráfico de mujeres: notas sobre la "economía política" del sexo. En $M$. Lamas, El Género: La construcción cultural de la diferencia del sexo (pp. 35-96). México: Porrúa/PUEG.

Ryle, G. (1949/2005). El Concepto de lo mental. Barcelona: Paidos.

Sabo, D. (2000). Comprender la salud de los hombres. Hardvard: OPS/Harvard Center for Population and Development Studies.

Scott, J. (1986/1996). El Género: una categoría útil para el análisis histórico. En M. Lamas, El Género: La construcción cultural de la diferencia sexual. (pp. 265-302). México: Porrúa/PUEG.

Stoller, J. (1968). Sex and Gender: On the development of masculinity and feminity. New York: Science House.

West, C., \& Zimmerman, D. (1999). Haciendo Género. En M. Navarro, \& C. Stimpson, Sexualidad, Género y Roles Sexuales (pp. 109-143). Buenos Aires: FCE.

Williams, J. E., \& Best, D. L. (1990). Measuing sex stereotypes: A multination study. Newbury Park: Sage Publications.

Auto-referencias de autores: 0

Auto-referencias de revista JBHSI: 0 